\title{
A Clinical Study on Comprehensive Analysis of Enterocutaneous Fistula
}

\author{
Sanjeeva Raju Kunche ${ }^{1}$, A. Manoj², Chetan Kumar A. ${ }^{3}$
}

1Department of General Surgery, Sri Venkateshwaraa Medial College and Research Centre, Ariyur, Pondicherry, India. 2Department of General Surgery, Sri Venkateshwaraa Medial College and Research Centre, Ariyur, Pondicherry, India. ${ }^{3}$ Department of General Surgery, Sri Venkateshwaraa Medial College and Research Centre, Ariyur, Pondicherry, India.

\section{ABSTRACT}

\section{BACKGROUND}

An enterocutaneous fistula (ECF) is an abnormal communication between the small or large bowel and the skin. It is one of the serious complications of gastrointestinal surgery and has a mortality rate of up to $20 \%$. Management involves dealing with malnutrition, sepsis, metabolic derangement and electrolyte imbalance.

\section{METHODS}

This is a combined prospective and retrospective study conducted at SVMC \& RC from June 2017 to May 2019. Thirty patients with ECF were enrolled in this study. Patients with biliary, pancreatic and anal fistulas were excluded. Factors influencing spontaneous closure of fistula such as fistula characteristics, organ of origin, sepsis etc. were studied. Initial management for all patients was conservative with restoration of fluid and electrolyte imbalance, and were initially started on parenteral nutrition using dextrose $10 \%$, Astymin, Intralipid, human albumin and total parenteral nutrition (TPN).

\section{RESULTS}

Fistula closed spontaneously in 22 patients. Operative closure was done in 5. Death occurred in 3 (mortality rate- 10\%). Out of 30 fistulas, 28 were post-operative fistulas. One was due to trauma and one was due to Crohn's disease. Among the former, 83\% developed a fistula following emergency surgery. Albumin levels after 3 weeks of treatment, were predictive of both spontaneous closure and mortality.

\section{CONCLUSIONS}

Post-operative causes account for majority of enterocutaneous fistulas. Anatomical origin of fistula, associated factors like sepsis, malnutrition, hypoalbuminemia and abdominal wall defect, influence the spontaneous closure. Total parenteral nutrition increases the spontaneous closure rate of fistulas and decrease mortality rate.

\section{KEY WORDS}

Enterocutaneous Fistula, Intestinal Fistula, Bowel Fistula, Faecal Fistula
Corresponding Author: Dr. Sanjeeva Raju Kunche, No. 202, A-Block, $2^{\text {nd }}$ Floor, Sri Venkateshwaraa Medical College and Research Centre, Ariyur-605009, Pondicherry, India.

E-mail: sanjeev2001jipmer@yahoo.com

DOI: $10.14260 / j e m d s / 2019 / 818$

Financial or Other Competing Interests: None.

How to Cite This Article:

Kunche SR, Manoj A, Kumar CA. A clinical study on comphrehensive analysis of enterocutaneous fistula. J. Evolution Med. Dent. Sci. 2019;8(50):3775-3779, DOI: $10.14260 /$ jemds $/ 2019 / 818$

Submission 03-10-2019,

Peer Review 20-11-2019,

Acceptance 06-12-2019,

Published 16-12-2019. 


\section{BACKGROUND}

Enterocutaneous Fistula, one of the serious complications of gastrointestinal surgery, imposes a certain amount of chronic morbidity to the patient and has a mortality rate of up to $20 \%$. Management of enterocutaneous fistula patients involves team work especially for dealing malnutrition, sepsis, metabolic derangement and electrolyte imbalance. In spite of an immense amount of advancement in the post-operative care now a days they remain one of the fascinating challenges to the surgeon. While treating these patients it is important to know when to wait for a spontaneous closure and when to do a surgical closure. ${ }^{1}$ The word 'fistula' is derived and identical latin word for a pipe but its incorporation into English medical terminology was probably not directly from its Latin origin, but from the old French word 'Festre' which led to the English word 'Fistle' and 'Fistula'.2

A fistula is an abnormal communication between two epithelialized surfaces. An enterocutaneous fistula (ECF) is defined as an abnormal communication between the small or large bowel and the skin. Although fistulas arising from other regions of the gastrointestinal (GI) tract (e.g.: stomach, oesophagus) may sometimes be included in the definition of $\mathrm{ECF}$, the discussion is limited to the conventional definition of ECF. An ECF, which is classified as an external fistula (As opposed to an internal fistula, which is an abnormal communication between 2 hollow viscera), is a complication that is usually seen following surgery on the small or large bowel. ECF is a common presentation in general surgical wards, and despite advances in the management of these lesions, they are still responsible for a significant mortality rate, ranging from $5-20 \%$, due to associated sepsis, nutritional abnormalities, and electrolyte imbalances. Understanding the pathophysiology of, as well as the risk factors for, ECFs should help to reduce their occurrence. Moreover, the wellestablished treatment guidelines for these lesions, along with some newer treatment options, should help clinicians to achieve a better outcome in patients with an ECF. 3,4,5

The most commonly used schemes for classification are anatomic, physiologic and etiologic which are not exclusive of one another. Sitges- Sera et $\mathrm{al}^{5}$ proposed an alternative classification of postoperative ECF which integrates the role of nutrition, surgery and prognosis of a given patient and which can be used as a guideline to treatment.

Enterocutaneous fistula (ECF) is a common condition in most general surgical wards. Over the past few decades, improvement in the management of ECF has resulted in a gradual decrease in the associated mortality rate. Nonetheless, the mortality rate from these lesions, at $5-20 \%$, remains significant. Once a patient develops an ECF, the morbidity associated with the surgical procedure or the primary disease increases, affecting the patient's quality of life, lengthening his/her hospital stay, and raising the overall cost of treatment.

Treatment of enterocutaneous fistulas (ECFs) continues to be a difficult task. The problems associated with an intestinal wound breakdown were mentioned as early as $53 \mathrm{BC}$, by Celsus, who stated that the large intestine can be sutured, not with any certain assurance, but because this doubtful hope is preferable to certain despair; for occasionally it heals up." In the mid-19th century, John Hunter also described the difficulties in treating ECFs, insisting that "in such cases nothing is to be done but dressing the wound superficially, and when the contents of the wounded viscus become less, we may hope for a cure." In a landmark article, Edmunds et al provided a comprehensive discussion of ECF. Out of 157 patients in the study, 67 developed ECF following surgery. Important complications of ECF included fluid and electrolyte imbalance, malnutrition, and generalized peritonitis. Mortality was $62 \%$ in patients with gastric and duodenal fistulas, $54 \%$ in patients with small-bowel fistulas, and $16 \%$ with colonic fistulas.

Enterocutaneous fistulas can occur as a complication following any type of surgery on the GI tract. Indeed, more than $75 \%$ of all ECFs arise as a postoperative complication, while about $15-25 \%$ of them result from abdominal trauma or occur spontaneously in relation to cancer, irradiation, inflammatory bowel disease, or ischemic or infective conditions. Previous studies reported mortality rate as high as $65 \%$ in cases with ECF. With recent advances in radio imaging, nutritional support and effective antibiotic availability, now the mortality rate has been reduced to $20 \% .5,6$

We wanted to analyse all cases of ECF treated in our institute, to identify the factors influencing the spontaneous closure, mortality, presentation, prognosis, outcome of patients and assess the importance of management of fistula.

\section{METHODS}

This was a combined prospective and retrospective study conducted at the SVMC \& RC. Study was done after getting approval from the institutional ethical committee, and an informed consent was obtained from patients. The period of study was from June 2017 to May 2019. All patients with ECF were enrolled in this study. Patients with biliary, pancreatic and anal fistulas were excluded.

Initial management for all patients was conservative with restoration of fluid and electrolyte balance being given the first priority. All patients were initially started on parenteral nutrition using dextrose 10\%, Astymin, Intralipid, Human albumin and TPN. Later some were switched over to enteral or Ryles feeds depending on the clinical situation.

Patients with clinical features of sepsis were subjected to an ultrasound scan and treated with transperitoneal drainage if there was significant intra-abdominal collection. Emergency surgical intervention was undertaken only in patients who had an intra-abdominal abscess. Diversion procedure were undertaken in some patients at this time. Elective surgical closure was done if the fistula failed to close spontaneously after 4 weeks of conservative management. A proforma was made and all cases of enterocutaneous fistulas were followed up from the day of recognition of fistula and till the date of discharge. Details regarding types of surgery performed, category of the fistula, origin of fistula were collected. Management policies for each patient is individualized and details of management were taken down. Analysis of morbidity and mortality were taken into the study.

\section{Statistical Analysis}

The statistical analysis was done by using SPSS software version 10.0 was used. $p$ Value of less than $5 \%$ was considered as significant. 


\section{RESULTS}

A total of 30 patients were studied. There were 23 males $(77 \%)$ and 7 females (23\%) involved in this study. Males were commonly affected compared to females, 23 males $>7$ females. Majority of the fistulas were observed in the age group of 30 to 60 years. Out of 30 fistulas, 28 were post-operative, 1 was due to trauma and 1 was due to Crohn's disease. Among the former group $83 \%$ developed a fistula following emergency surgery. The mean interval between the initial surgery and appearance of the fistula was 9 days. Ileal fistulas were the most common accounting for $46.66 \%$ of patients (refer- table-1). Among the surgeries responsible for ECF, perforation closure accounted for the maximum in 22 (73.3\%) patients (Refer- Table- 2).

Out of 30 patients, $11(36.6 \%)$ patients had high output and $19(63.3 \%)$ had low output fistulas (Refer -Table-3). Fistulas closed in 22 patients spontaneously, operative closure was done in 5 patients. Death occurred in 3 patients with a mortality rate of $10 \%$ (Refer- Table- 4 ).

\begin{tabular}{|c|c|}
\hline Site of Origin of ECF & No. of Patients \\
\hline Duodenum & 8 \\
\hline Jejunum & 1 \\
\hline Ileum & 14 \\
\hline Colon and Appendix & 7 \\
\hline \multicolumn{2}{|c|}{ Table 1. Based on Site of ECF Involved } \\
\hline
\end{tabular}

\begin{tabular}{|c|c|}
\hline Types of Surgery & No. of Patients \\
\hline Perforation closure & 22 \\
\hline Intestinal resection and anastomosis & 6 \\
\hline Appendicectomy & 1 \\
\hline Others & 1 \\
\hline \multicolumn{2}{|c|}{ Table 2. Based on Surgeries Causing ECF } \\
\hline
\end{tabular}

\begin{tabular}{|c|c|c|}
\hline Site of Fistula & \multicolumn{2}{|c|}{ No. of Patients } \\
\hline & High Output & Low Output \\
\hline Duodenum & 5 & 3 \\
\hline Jejunum & - & 1 \\
\hline Ileum & 5 & 9 \\
\hline Colon & 1 & 5 \\
\hline Appendix & - & 1 \\
\hline \multicolumn{2}{|c|}{ Table 3. Based on Output of Fistula Involved } \\
\hline
\end{tabular}

\begin{tabular}{|c|c|}
\hline Outcome & No. of Patients \\
\hline Closed by surgical intervention & 5 \\
\hline Spontaneous closure & 22 \\
\hline Death & 3 \\
\hline \multicolumn{2}{|c|}{ Table 4. Based on the Outcome of Patients with ECF } \\
\hline
\end{tabular}

\begin{tabular}{|c|c|c|c|c|}
\hline Risk Factors & No. of Patients & Closure & Closure & Expired \\
\hline & & Spontaneous & Operative & \\
\hline Age $>51$ years & 15 & 9 & 4 & 2 \\
\hline Sepsis & 14 & 6 & 5 & 3 \\
\hline Malnutrition & 15 & 7 & 5 & 3 \\
\hline Large abdominal defect & 9 & 1 & 5 & 3 \\
\hline High output & 11 & 3 & 5 & 3 \\
\hline Serum Albumin <3 gm \% & 9 & 1 & 5 & 3 \\
\hline \multicolumn{6}{|l}{ Table 5. Based on Risk Factors Involved } \\
\hline
\end{tabular}

Patients with single or no risk factor had no mortality refer (Table-5), whereas mortality steadily rose as risk factors accumulated. The mean duration of the fistula closure was achieved in 32 days in case of spontaneous closure.

Among the risk factors analysed the presence of a large abdominal wall defect and high output fistulas were significantly associated with failure of the fistula to close spontaneously. Among the factors analysed with respect to survival hypoalbuminemia, malnutrition and sepsis were significantly associated with high mortality.

Serum albumin levels measured at the beginning of treatment showed no significant difference between patients who had spontaneous closure and those who did not, but there was a significant difference in serum albumin levels at the beginning of treatment between survivors and non survivors.

\section{Effects of TPN}

- $\quad$ TPN increases the spontaneous closure rate of fistula.

- TPN reduces the duration of fistula by hastening the spontaneous closure.

- $\quad$ TPN has contributed to decreased mortality in patients with fistula.

- Even if spontaneous closure is not achieved because of complex fistula anatomy the patient's general condition was improved to be taken up for definitive surgery.

\section{DISCUSSION}

The outcome of treatment of enterocutaneous fistulas vary widely from hospital to hospital. Mortality rates quoted in literature varies from $6.25 \%$ to $60 \%$. Spontaneous closure occurs in 30 to $70 \%$. This is because of the heterogenous nature of this condition. This study revealed a mortality rate of $10 \%$ and spontaneous closure rate of $73.33 \%$ which is similar to other studies in literature. Ileal fistulas in this study closed spontaneously in $92.3 \%$ cases, which is far better compared to other series where the rates are less than $40 \%$ despite it being an anatomically favourable location. In this study Ileal fistulas were the most common accounting for $46.66 \%$ of patients. A similar distribution is reported in a study done by Roback et al. ${ }^{7}$

In a study done by Edmunds et $\mathrm{al}^{1}$ out of 157 patients studied, 67 developed ECF following surgery. Important complications of ECF included fluid and electrolyte imbalance, malnutrition, and generalized peritonitis. Mortality was $62 \%$ in patients with gastric and duodenal fistulas, $54 \%$ in patients with small-bowel fistulas, and $16 \%$ with colonic fistulas. Reber et al ${ }^{8}$ observed that $90 \%$ of patients who had closure of fistula did so within 1 month after sepsis was controlled. Less than $10 \%$ closed in 2 months and none after 3 months. Fazio et al $^{9}$ observed that surgical mortality and success rate when surgery was done before and after 6 weeks was 21 and $70 \%$ and 12 and $84 \%$ respectively. Thus, elective surgery is best undertaken at least 4 to 6 weeks after resolution of intraabdominal sepsis, which is usually $60-75$ days after initial surgery.

In this study among the factors analysed the only factor that was predictive of spontaneous closure was the size of the abdominal wall defect. It was found that malnutrition, hypoalbuminemia and large abdominal wall defect were all predictive of mortality. Altomare et al showed that sepsis, serum albumin and high output fistulas were significantly related to the risk of death in ECF, which is similar to our study.

Operative closure was undertaken between 4 to 6 weeks except in one case where it was done in the first week for emergency drainage of an intra-abdominal abscess. In the 22 patients who had spontaneous closure only 6 had sepsis which was controlled by giving higher antibiotics, 7 had malnutrition 
which was corrected and 1 had abdominal wall defect. None of them had hypoalbuminemia. Mortality in this series was due to a combination of risk factors with the rate increasing as these factors accumulated. Among the 5 cases that needed operative closure all cases had sepsis, 3 had abdominal wall defect, 1 patient had distal obstruction and abdominal wall defect and one had Crohn's disease. Although serum albumin levels at the appearance of the fistula did not predict spontaneous closure, it was predictive of mortality. Kuvshinoff et al ${ }^{10}$ reported that serum transferrin levels of $>200 \mathrm{mg} / \mathrm{dl}$ was predictive of spontaneous closure. Age of the patient influences mortality, but not spontaneous closure. Rubelowsky et al ${ }^{11}$ reported spontaneous closure rates have been estimated to be between $50 \%$ and $80 \%$.

In this study the albumin levels after 3 weeks of treatment, however, were predictive of both spontaneous closure and mortality. This shows that improving the nutritional status of the patient as evidenced by the increase in serum albumin levels after 3 weeks of treatment increases the chances of spontaneous closure. Similarly, failure to correct malnutrition as evidenced by decreased serum albumin levels after 3 weeks increases the mortality. Altomare et al reported a statistically significant difference in albumin levels between survivors and non-survivors and concluded that serum albumin levels have a $94 \%$ accuracy in predicting outcome. In this study another outcome is TPN which increases the spontaneous closure and also decreases the mortality in enterocutaneous fistula. Coutsoffides $^{12}$ reported mortality rates of $32 \%$ and $4 \%$ in the malnourished and well-nourished patients respectively. Serum visceral protein levels can be used to predict outcome in ECF.

This study revealed a mortality rate of $10 \%$ and spontaneous closure rate of $73.33 \%$ which is similar to other studies in literature. Edmunds, Sitges Serra et al and Levy observed mortality rates of $54 \%, 32 \%$ and $50 \%$ for high output fistulas and $16 \%, 6 \%$ and $26 \%$ for low output fistulas respectively. Chapman ${ }^{13}$ observed a mortality rate of $12 \%$ and spontaneous closure in $89 \%$ of patients with ECF who received more than $3000 \mathrm{kcal} / 24 \mathrm{hrs}$. But in patients who received less than $1000 \mathrm{kcal} / 24 \mathrm{hrs}$ corresponding values were 55\% and $37 \%$. Fazio et al $^{9}$ reported mortality rate of $0 \%$ and $42 \%$ in patients with serum albumin above $3.5 \mathrm{~g} / \mathrm{dl}$ and below $2.5 \mathrm{~g} / \mathrm{dl}$ respectively. Reber et al ${ }^{8}$ reported mortality rates of $22 \%$ and $48 \%$ in patients below and above 65 years respectively. Narsos et al ${ }^{14}$ reported a mortality rate of $48 \%$ for jejunal fistulas and $18 \%$ for ileal fistulas in their study. Hollender's et al $^{15}$ in their study reported that advances in percutaneous drainage of abscesses and the availability of better antibiotics could be responsible for the huge difference in mortality reported.

In the present study, $73 \%$ of the total patients were managed conservatively, whereas $27 \%$ of patients required surgical intervention. The common surgeries done in our patients are resection of segment of bowel containing fistula and end-to-end anastomosis, exteriorization of both ends, adhesiolysis and primary closure. Our study correlates with Lorenzo et $\mathrm{al}^{16}$ and Tarazi et $\mathrm{al}^{17}$ that the timing of operation and selection of the operative procedure depends on the type of fistula, response to conservative management, and the state of the patient.

\section{CONCLUSIONS}

Post-operative causes account for majority of enterocutaneous fistulas. Anatomical origin of fistula, associated adverse factors like sepsis, malnutrition, hypoalbuminemia and abdominal wall defect influence the spontaneous closure. Total parenteral nutrition increases the spontaneous closure rate of fistulas and also decreases of mortality rate. Definitive surgery should be carried out in the following patients

- Spontaneous closure not occurred even after 4 to 6 weeks of conservative therapy.

- Patient having complex fistula anatomy.

- Patient having distal bowel obstruction.

The management of ECF needs competent teamwork. The team members are the surgeon, staff nurses, stoma therapist, physiotherapist, dietician and pharmacist.

\section{REFERENCES}

[1] Edmunds LH Jr, Williams GH, Welch CE. External fistulas arising from the gastro-intestinal tract. Ann Surg 1960;152:445-71.

[2] Fischer JE. The pathophysiology of enterocutaneous fistulas. World J Surg 1983;7(4):446-50.

[3] Scott MB, Josef EF. Bilary and gastrointestinal fistulas. In: Michael JZ, Seymour IS, Harold E, eds. Maingot's Abdominal Operations. $10^{\text {th }}$ edn. Stamford: Appleton \& Lange 1997: p. 581-93.

[4] Berry SM, Fischer JE. Classification and pathophysiology of enterocutaneous fistulas. Surg Clin North Am 1996;76(5):1009-18.

[5] Sitges-Serra A, Jaurrieta E, Sitges-Creus A. Management of postoperative enterocutaneous fistulas: the role of parenteral nutrition and surgery. $\mathrm{Br} \quad \mathrm{J}$ Surg 1982;69(3):147-50.

[6] McIntyre PB, Ritchie JK, Hawley PR, et al. Management of enterocutaneous fistulas: a review of 132 cases. Br J Surg 1984;71(4):293-6.

[7] Roback SA, Nicoloff DM. High output enterocutaneous fistulas of the small bowel. An analysis of fifty-five cases. Am J Surg 1972;123(3):317-22.

[8] Reber HA, Roberts C, Way LW, et al. Management of external gastrointestinal fistulas. Ann Surg 1978;188(4):460-7.

[9] Fazio VW, Coutsoftides T, Steiger E. Factors influencing the outcome of treatment of small bowel cutaneous fistulas. World J Surg 1983;7(4):481-8.

[10] Kuvshinoff BW, Brodish RJ, McFadden DW, et al. Serum transferrin as a prognostic indicator of spontaneous closure and mortality in GI cutaneous fistulas. Ann Surg 1993;217(6):615-23.

[11] Rubelowsky J, Machiedo GW. Reoperative versus conservative management for gastrointestinal fistulas. Surg Clin North Am 1991;71(1):147-57.

[12] Coutsoftides T, Fazio VW. Small intestine cutaneous fistulas. Sur Gynaecol Obstet 1979;149(3):333-6.

[13] Chapman R, Foran R, Dunphy JE. Management of intestinal fistulas. Am J Surg 1964;108(2):157-64. 
[14] Nassos TP, Braasch JW. External small bowel fistulas. Surg Clin North Am 1977;51:687-92.

[15] Hollender LF, Meyer C, Avet D, et al. Postoperative fistulas of the small intestine: therapeutic principles. World J Surg 1983;7(4):474-80.
[16] Lorenzo GA, Beal JM. Management of external small bowel fistulas. Arch Surg 1969;99(3):394-6.

[17] Tarazi R, Coutsoftides T, Steiger E, et al. Gastric and duodenal cutaneous fistulas. World J Surg 1983;7(4):46373. 